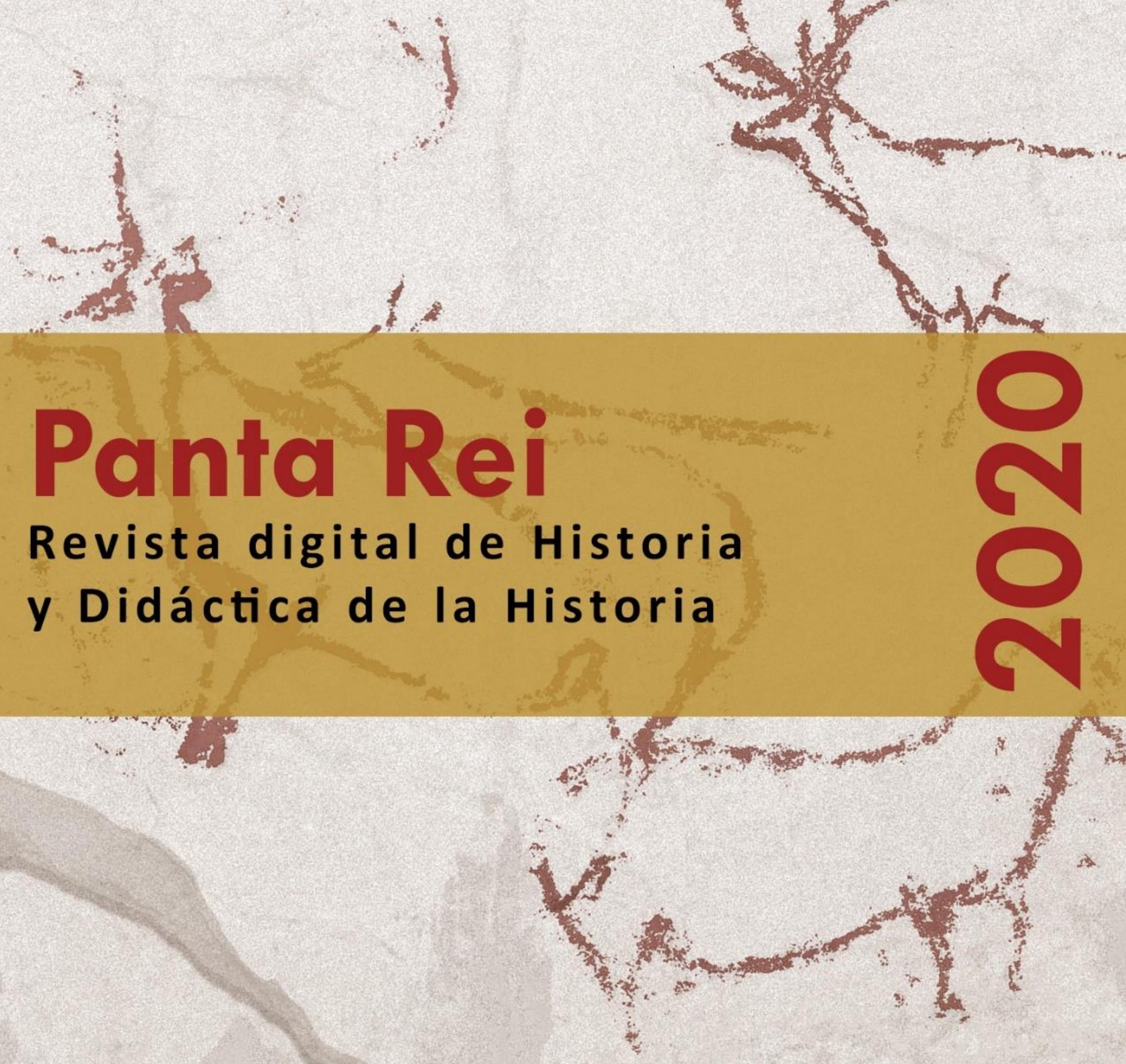




\section{0}

Revista anual

Fecha de inicio: 1995

RevistaPantaRei.pantarei@um.es

\section{Edita:}

Centro de Estudios del Próximo Oriente y la

Antigüedad Tardía - CEPOAT

Edificio Universitario Saavedra Fajardo.

Universidad de Murcia

C/ Actor Isidoro Máiquez, 9

30007 - MURCIA - ESPAÑA

Teléfono: $(+34) 868883890$

cepoat@um.es

Web: www.um.es/cepoat/pantarei

Ediciones de la Universidad de Murcia - EDITUM

Edificio Pleiades. Campus de Espinardo.

Universidad de Murcia

$\mathrm{C} /$ Campus, s/n

30100 - MURCIA - ESPAÑA

Teléfono: $(+34) 868883013$

En portada: calco de las pinturas rupestres de la editum@um.es

Web: https://www.um.es/web/editum/

Cueva del Niño. García Moreno et al., 2016.

Edición 2020

ISSNe: 2386-8864

Responsables de los textos: sus autores.

ISSN: $1136-2464$

Depósito legal: MU-966-1995

Responsable de la presente edición: Consejo Editorial de Panta Rei. 


\section{CONSEJO DE REDACCIÓN}

Coordinador editorial

Egea Vivancos, Alejandro [Didáctica de las Ciencias Sociales, Universidad de Murcia]

Secretaria

Arias Ferrer, Laura [Didáctica de las Ciencias

Sociales, Universidad de Murcia]

Editores

Jiménez Vialás, Helena [Historia Antigua, Universidad de Murcia]

Martínez Gil, Tània [Didáctica de las Ciencias

Sociales, Universidad de Barcelona]

Meseguer Gil, Antonio José [Historiador, Profesor de

Secundaria]

Ortiz García, Jónatan [Arqueología, Universidad de

Alcalá de Henares]

Romero Molero, Alberto [Arqueología, Universidad

Isabel I]

Sáez Giménez, David Omar [Historiador, Profesor

de Secundaria]

Sáez Rosenkranz, Isidora V. [Didáctica de las

Ciencias Sociales, Universidad de Barcelona]

Sánchez Mondejar, Celso Miguel [Arqueólogo,

Patrimonio Inteligente]

Responsable informático

Martínez García, José Javier [CEPOAT, Universidad de Murcia]

Responsables de traducción y corrección lingüística Martínez Martínez, Cristina [Profesora de Secundaria, Sociedad Española de Lenguas Modernas] Albaladejo Albaladejo, Sara [ISEN-Universidad de Murcia]

\section{CONSEJO ASESOR}

Adroher Auroux, Andrés María [Arqueología, Universidad de Granada]

Albero Muñoz, $M^{a}$ del Mar [H. ${ }^{a}$ del Arte, Universidad de Murcia]

Alia Miranda, Francisco [Historia Contemporánea, UCLM]

Arciniega García, Luis [Historia del Arte, Universidad de Valencia]

Barrio Barrio, Juan Antonio [Historia Medieval,

Universidad de Alicante]

Castellano i Solé, Núria [Egiptología, Schola

Didàctica Activa S.L.]

Chapman, Arthur [History Education, University

College of London, Reino Unido]

Cid López, Rosa María [Historia Antigua, Universidad de Oviedo]

Cobacho López, Ángel [Derecho, Universidad de Murcia]

Cuenca López, José María [Didáctica de las Ciencias Sociales, Universidad de Huelva]
Egea Bruno, Pedro M. ${ }^{a}$ [Historia Contemporánea, Universidad de Murcia]

Feijoo Martínez, Santiago [Arqueología, Consorcio

Ciudad Monumental de Mérida]

García Atienzar, Gabriel [Prehistoria, Universidad de

Alicante]

Ginestí Rosell, Anna [Filología Clásica, Katholische

Universität Eichstätt-Ingolstadt]

González Monfort, Neus [Didáctica de las

Ciencias Sociales, Universidad Autónoma de

Barcelona]

González Soutelo, Silvia [Arqueología, Universidad de Vigo]

Haber Uriarte, María [Prehistoria, Universidad de Murcia]

Hernández de la Fuente, David [Filología Clásica, Universidad Complutense]

Hutson, Scott R. [Anthropology, University of Kentucky, EEUU]

Igual Luis, David [Historia Medieval, UCLM]

Irigoyen López, Antonio [Historia Moderna,

Universidad de Murcia]

Jover Maestre, Francisco Javier [Prehistoria,

Universidad de Alicante]

Mahony, Simon [Digital Humanities, University College of London, Reino Unido]

Marsilla de Pascual, Francisco Reyes [Técnicas

historiográficas, Universidad de Murcia]

Martínez-Burgos García, Palma [H. ${ }^{a}$ del Arte, UCLM]

Mathis, Christian [Didaktik der Geschichte, PH Zürich]

Miralles Maldonado, José Carlos [Filología Clásica,

Universidad de Murcia]

Molina Gómez, José Antonio [Historia Antigua,

Universidad de Murcia]

Mónica Ghirardi [Historia Moderna, Universidad

Nacional de Córdoba, Argentina]

Navarro Espinach, Germán [Historia Medieval,

Universidad de Zaragoza]

Noguera Celdrán, José Miguel [Arqueología,

Universidad de Murcia]

Ortiz Heras, Manuel [Historia Contemporánea, UCLM]

Panzram, Sabine [Historia Antigua, Universität

Hamburg]

Pérez Molina, Miguel Emilio [Filología Clásica,

Universidad de Murcia]

Prados Martínez, Fernando [Arqueología,

Universidad de Alicante]

Sánchez lbáñez, Raquel [Didáctica de las Ciencias

Sociales, Universidad de Murcia]

Sancho Gómez, Miguel Pablo [Educación, UCAM]

Victoria Moreno, Diego [Historia Contemporánea,

UNED]

Vilar García, María José [Historia Contemporánea,

Universidad de Murcia]

Vivas Sainz, Inmaculada [H. ${ }^{a}$ del Arte, UNED]

Zamora López, José Ángel [Próximo Oriente Antiguo,

CCHS-CSIC] 



\section{Índice}

\section{Artículos}

Las ocupaciones paleolíticas en el sur de la provincia de Albacete

Noelia Sánchez Martínez

El elefante en las acuñaciones hispanocartaginesas

José Luis Aledo Martínez

La epigrafía votiva romana de Caldas de Montbui (Vallés Oriental, Barcelona) (ss. I-II d. C.). Un ejemplo de promoción de las élites provinciales de la tarraconensis en centros de aguas mineromedicinales

Jesús Sánchez Alguacil

Representación de la historia de España por medio de la filatelia. Estudio de los sellos diseñados por Gallego y Rey

Pedro Vázquez-Miraz

La representación del patrimonio arqueológico en los libros de texto de Educación Primaria: EI 109 contexto indígena canario como estudio de caso

A. José Farrujía, Carmen Ascanio Sánchez, Ulises Martín Herández y Cristo Manuel Hernández Gómez

La empatía como elemento para la adquisición del pensamiento histórico en alumnos de 129 bachillerato. Un estudio de caso centrado en la Guerra Civil española y el franquismo Sebastián Molina Puche y Adrián Salmerón Ayala

Fuentes orales para el desarrollo de la empatía histórica: un estudio en la formación del 155 profesorado de Educación Infantil

$M^{a}$. Teresa Carril-Merino, Beatriz Andreu-Mediero, Mercedes de la Calle Carrecedo y Esther López Torres

¿Qué aporta el género a la formación de docentes de historia? La valoración de la perspectiva de género entre los estudiantes del Máster de Profesor/Profesora en Educación Secundaria Helena Rausell Guillot

Educar para una ciudadanía crítica: una investigación a partir de los usos y finalidades de la historia escolar

Néstor Banderas Navarro

\section{Reseñas}

A. Brilli (2018). El viaje a Oriente, Madrid: A. Machado Libros, 390 págs Juan Álvarez García

Altamira (Hugh Hudson, 2016) 



\title{
¿Qué aporta el género a la formación de docentes de historia? La valoración de la perspectiva de género entre los estudiantes del Máster de Profesor/Profesora en Educación Secundaria ${ }^{1}$
}

\author{
Does Gender Matter while Teaching Training in History? Gender \\ Perspective Assessment by Master's Degree in Secondary Education \\ Teaching Students
}

Helena Rausell Guillot Universidad de Valencia

Helena.rausell@uv.es

0000-0003-1803-3487

Recibido: $19 / 11 / 2019$

Aceptado: 04/06/2020

\begin{abstract}
Resumen
Nuestro artículo presenta los resultados de una investigación sobre género e historia enseñada diseñada e implementada mediante cuestionarios en el Master de Formación de Profesor/Profesora de Enseñanza Secundaria de la Universidad de Valencia (especialidad de Geografía e Historia, cursos 2016-2017 y 2017-2018). Hemos tratado de determinar tanto la formación previa en género de los y las participantes como la valoración que les merecen las aportaciones realizadas por la perspectiva de género a la enseñanza de la historia y su investigación. Los cuestionarios han sido completados por una muestra de 70 estudiantes. Se trata de un estudio colectivo de casos en cuyo análisis hemos considerado como variables tanto la formación previa como el género de los entrevistados.
\end{abstract}

\section{Palabras clave}

Cuestiones de Género, Enseñanza de la Historia, Formación del profesorado, Identidad sexual, Investigación educativa.

\begin{abstract}
This article assesses trainee teachers' appraisal of women history and socially relevant subjects as gender. The aim is to evaluate how participants value gender into teaching and learning processes and in regard to history teaching and research. Thus, discourse and statements related to gender have been analysed and categorized by means of initial surveys. The sample consists of 70 students from the Master's degree in Secondary Education Teaching (University of Valencia, years 2016-2017 and 2017-2018, majoring in Geography and History). Results have been discussed according to the previous gender training and they also take into account gender bias.
\end{abstract}

\section{Keywords}

Gender Issues, History Instruction, Teacher Education, Sexual identity, Educational Research.

Para citar este artículo: Rausell Guillot, H. (2020). ¿Qué aporta el género a la formación de docentes de historia? La valoración de la perspectiva de género entre los estudiantes del Máster de Profesor/Profesora en Educación Secundaria. Panta Rei: revista digital de Historia y didáctica de la Historia, 185-203. doi: 10.6018/pantarei.444781

\footnotetext{
${ }^{1}$ Dedicado al Dr. Joan Pagès Blanch, in memoriam.
} 
"La mente no tiene sexo". (Poullain de la Barre, en Schiebinger, 2004, p. 11).

\section{Introducción}

El género parece haber llegado para quedarse. Desde sus inicios en los años 70 del siglo XX, aparece como una corriente de escritura, renovación y enseñanza de los saberes en general y de la historia en particular que añade a su transversalidad un enorme potencial transformador. No sin dificultades, ha conseguido abrirse un hueco relativamente importante en la docencia y en la investigación universitaria, aunque la situación no es la misma en los niveles no universitarios. En 2018, Bolufer realizaba un balance de la presencia del género en la enseñanza universitaria de la historia -con denominaciones como "historia de las mujeres", "historia del género" o "historia de las relaciones de género"- en distintos grados y post-grados universitarios a escala nacional. Llegaba a documentarla hasta en 28 universidades, 34 grados y 27 masters. Aunque se trata mayoritariamente de grados de historia (21, concretamente), estas materias también aparecen en algunos grados de historia del arte, historia y ciencias de la música, humanidades e incluso medicina o comunicación audiovisual. La Universidad de Valencia fue - junto a las de Barcelona, Granada, Oviedo, Complutense y Autónoma de Madrid - uno de los centros pioneros en su introducción, gracias a lo cual cuenta ya con una trayectoria de más de dos décadas (Bolufer, 2018).

\section{Marco teórico}

\subsection{El valor de introducir el género en la investigación}

La investigación en perspectiva de género se ha visto crecientemente respaldada en los últimos años por toda una serie de disposiciones autonómicas, estatales y supraestatales. Instituciones como el Parlamento Europeo han establecido en el Proyecto Horizonte 2020 el equilibrio de género en los equipos de investigación y en la toma de decisiones, la integración de la dimensión de género en la investigación y la innovación de contenido e incluso la posibilidad de pedir ayudas para la formación en materia de género como parte de los costes de financiación de los proyectos de investigación. Estas políticas responden a una realidad social, urgente y cambiante, que demanda a los y las docentes, políticos y políticas, investigadores e investigadoras, una mayor adecuación al contexto social y un mayor rigor en las investigaciones científicas. No en vano, distintas voces denuncian desde hace años que investigar sin integrar el análisis de sexo y género cuesta vidas y dinero (Schiebinger, 2004, 2008 y 2018). Evidencian, igualmente, el sesgo sexista de las investigaciones convencionales en elementos como el androcentrismo, la sobregeneralización (masculina), la insensibilidad de género, la existencia de dobles estándares, la dicotomía sexual o el familismo (Eichler, 1991).²

Aunque creciente, la investigación en género y didáctica de la historia sigue siendo escasa (Schneuwly, 2015). Nuestras disciplinas distan aún de entender el género como una cuestión central dentro de la problemática escolar o como un posible elemento vertebrador para la didáctica de la historia (Opériol, 2013). Son especialmente numerosos los estudios sobre género y materiales curriculares (Garreta y Careaga, 1987; Blanco, 2000; López-Navajas, 2014;

2 https://eur-lex.europa.eu/legal-content/ES/TXT/?uri=LEGISSUM\%3A2701 3.

Véase, igualmente https://genderedinnovations.stanford.edu/ 
Rugna, 2014; por destacar solo algunos). También, aunque son más escasas, existen propuestas didácticas para introducir la perspectiva de género en el aula de historia, geografía e historia del arte (Fernández, 2001; Hidalgo, Juliano, Roset y Caba, 2003) o a partir de enfoques transdisciplinares (Rausell y Talavera, 2019). Algunos de estos estudios denuncian la ausencia de las mujeres como sujetos en la historia enseñada, además de sugerir, especialmente desde el ámbito anglosajón, distintas posibilidades para incorporar a las mujeres al currículo de historia (Crocco, 1997; Lerner, 1979 y 1993; Mclntosh, 1983; Pagès y Sant, 2012; Woyshner, 2002), a lo que se añaden las muchas aportaciones del feminismo a la historia del arte (Alario, 2008; Hustvedt, 2017; López- F. Cao, 2001; Nochlin, 1989). Menor peso porcentual poseen, sin embargo, los trabajos que versan sobre la formación del profesorado en perspectiva de género, en consonancia con la escasa formación que los futuros docentes de historia, geografía e historia del arte reciben acerca de esta cuestión (Alonso, 1998; Crocco, 2006; Crocco y Cramer, 2005; Crocco y Libresco, 2007; Vavrus, 2009).

\subsection{Las aportaciones del género a la enseñanza de la historia}

Tradicionalmente nuestras sociedades han privilegiado una tradición de conocimiento y de comprensión centrada en el hombre que convierte a una minoría dominante en un grupo inclusivo, normativo e ideal para la humanidad (Minnich, 1990). En el caso de la historia, esto se hace evidente en la prevalencia de un relato tradicional de corte androcéntrico en el que los hombres parecen ser los únicos agentes posibles y en el que la inclusión de las mujeres tiende a producirse en relación con la lógica de la excepcionalidad (Fernández, 2001; Rausell, 2019). Esto estaría en conexión con la propia noción de "sujeto", según la aproximación de Foucault (1990), no como un fundamento o una estructura psicológica universal y atemporal, sino como una forma de subjetividad históricamente configurada a través de prácticas sociales y culturales en transformación constante y en relación directa con las distintas formas de poder.

Entre las aportaciones que ha realizado la historia de las mujeres a la enseñanza de la historia, podríamos destacar su contribución al desarrollo del pensamiento histórico (historicidad de las categorías de femenino y masculino), el valor otorgado a modelos no hegemónicos, minoritarios - discordantes, la necesidad de enriquecer la reflexión sobre la complejidad de las relaciones y las desigualdades sociales, la atención prestada a las formas variadas de la presencia y protagonismo de las mujeres en la historia o la crítica en torno a la aproximación esencialista que presenta la desigualdad entre hombres y mujeres como una diferencia de naturaleza, universal e invariable (Bolufer, 2018; Morant, 2017; Morant, Segura, Di Febo y Perry, 2013). A ello se añade la propia aparición del concepto de "género", que ha ido dotándose con el tiempo de nuevos contenidos y significados. En un primer momento se entendía el género como una herramienta analítica que permitía explicar la construcción social y cultural de la diferencia sexual (Scott, 1990 y 2008), como uno de los ejes primordiales de organización y jerarquización de las sociedades o como un sistema significante que estructura las categorías de pensamiento y las visiones del mundo (Bourdieu, 1997). Igualmente, en su relación con el sexo (Beauvoir, 2005; Butler, 1999) o a partir de sus vínculos con el poder (Butler, 2006; Foucault, 1990 y 2005). Con posterioridad, la reinterpretación del género desde la teoría queer ha pasado a definirlo como una construcción cultural que prima sobre las configuraciones corporales, de carácter no estático y que se materializa en una serie de actos performativos (Butler, 2006), como una "tecnología" (De Lauretis, 1989) en constante proceso de producción - reproducción (Mouffe, 1993), que se 
entiende en la intersección con otras categorías como raza, clase, edad u orientación sexual (De Lauretis, 1989; Witting, 2006).

La adopción del género dentro de la historia ha significado una ampliación de los temas de investigación (y de docencia) hasta incluir cuestiones como las relaciones familiares, los modelos y prácticas de la educación moral y sentimental o el análisis de los diferentes discursos sobre hombres y mujeres. Igualmente, la forma en la que el género expresa una relación de poder, situándonos dentro de un sistema jerárquico que realiza una valoración diferencial de los sujetos y de sus acciones y que formula siempre de manera implícita o explícita una intolerancia hacia la transgresión de las identidades sexuales normativas. A ello se añade la propia evolución de la historia de las mujeres, de tal modo que si, en un primer momento, su objetivo era visibilizar a las mujeres del pasado y reivindicarlas como sujetos históricos, pronto pasó también a analizar la forma en la que la dialéctica entre masculino y femenino actuaba como elemento clave en la construcción de las identidades y como condicionante de las acciones humanas. Más recientemente, la historia del género se ha aproximado al estudio de las masculinidades, esto es, la construcción de género de los sujetos varones o al género en su dimensión política, analizando las formas de participación de las mujeres en el poder y reivindicando también para ellas el espacio público (Aresti, 2010; Rose, 2010; Scott, 1990). Por esta razón, no puede entenderse el género sin la denuncia de las desigualdades y sin su conexión con el feminismo o, lo que es lo mismo, con el cambio social. En nuestra opinión, existe una imbricación entre la aparición de los denominados gender studies y la profunda transformación que las sociedades occidentales han vivido en el siglo XX y los primeros decenios del siglo XXI, la consolidación del movimiento feminista y la contestación a las formas de sometimiento y discriminación que han sido aplicadas sobre las mujeres y sobre las identidades de género no normativas, hasta tal punto que resulta difícil pensar el género sin la dimensión social y política que ha permitido su conceptualización (Bereni, Chauvi, Jaunat y Revillard, 2008).

\section{Metodología y fuentes}

\subsection{La metodología cualitativa y el diseño de los instrumentos de recogida de resultados. El establecimiento de las categorías}

Para llevar a cabo nuestra investigación hemos diseñado una serie de cuestionarios que, aplicados en distintos momentos a lo largo de dos cursos sucesivos (2016-2017 y 2017-2018), pudieran permitirnos conocer mejor el grado de formación en materia de género de los futuros docentes de historia, además de su valoración de las aportaciones que la perspectiva de género ha realizado dentro de la historia y las restantes ciencias sociales. En su diseño hemos considerado algunas de las etapas necesarias para la adopción del género en la investigación, identificadas por la filósofa feminista Sandra Harding (1987), entre ellas: la incorporación de las mujeres al estudio; el análisis de sus puntos de vista; la evidencia del sexismo que viene entremezclado con el saber tradicional; la formulación de teorías que expliquen la realidad incluyendo la perspectiva de género o el enunciado de teorías más rigurosas, de mayor nivel explicativo y con un componente transformador.

La investigación se enmarca dentro de enfoques de tipo cualitativo y en relación tanto con la teoría crítica como con el feminismo. Está enfocada a partir del estudio colectivo de casos (Simons, 2011 ; Stake, 2007). Cuestiona las finalidades de la enseñanza de la historia, la ausencia de las 
mujeres en su relato tradicional, la relevancia de la introducción de la perspectiva de género en el profesorado en formación, las posibilidades de incorporación de las mujeres al currículo o la propia noción de sujeto de la historia. Como indican Oakley (1998) y Lather (1991), el mero hecho de que el objeto de estudio tenga relación con la situación de las mujeres ya genera procesos de transformación que se inician al plantear el cuestionamiento de la propia realidad.

Para la recogida de la información, hemos utilizado el método de encuesta, que consiste en interrogar o preguntar sobre una gama de sucesos presentes, pasados o futuros a un conjunto de personas (Ruiz, 2009). Con este fin, hemos diseñado y/o implementado un total de tres cuestionarios (pre-test, intermedio y final o de auto-evaluación) que fueron aplicados en diferentes momentos de la investigación (cursos 2016-2017 y 2017-2018) a una muestra de 70 estudiantes del Máster Universitario de Formación en Profesor/Profesora de Enseñanza Secundaria de la Universidad de Valencia. Los resultados que aquí vamos a considerar están extraídos del primero de todos ellos, el cuestionario inicial, integrado por cinco preguntas abiertas.

Los cuestionarios respetan el anonimato de los y las participantes. En su confección hemos considerado una serie de variables, entre las que se incluyen el nivel de formación de los participantes, el género y la edad. Igualmente, se han incluido variables de control de los propios cuestionarios, en este caso, en relación al número de cuestionarios, de participantes o los propios datos del entrevistador. Por último, hemos valorado criterios como la temporalización, las condiciones de realización o el ciclo de muestreo (Alaminos y Castejón, 2006). Respecto a la variable género, hemos tratado de huir de la imposición del binario femenino - masculino, mujer - hombre, incluyendo la opción "otros", a fin de no presentar solo dos versiones del género, dadas imaginariamente por el cuerpo del individuo. Sin embargo, ninguno de los participantes ha marcado dicha opción en su respuesta.

\subsection{Pregunta(s) y objetivos de la investigación. Całegorización}

Los resultados que ahora presentamos están, obviamente, relacionados con una serie de preguntas, supuestos y objetivos de investigación. Dichas preguntas y objetivos se enmarcan dentro de un primer gran interrogante relacionado con los conocimientos que los futuros docentes de historia tienen del género y con su valoración como perspectiva o enfoque, a propósito de sus posibles aportaciones a la disciplina. Algunas de estas preguntas de investigación son:

- ¿Conocen los graduados de historia, geografía o historia del arte las aportaciones realizadas en las últimas décadas por la historia de las mujeres? ¿Han recibido algún tipo de formación en género?

- ¿̇Qué alternativas sugieren los participantes para incluir a las mujeres en la enseñanza de las ciencias sociales? ¿Cuáles consideran que son las limitaciones de excluir a las mujeres y su historia de la práctica del aula? ¿Cuáles serían según ellos las ventajas de incluirlas?

- ¿̇Son capaces de diseñar e implementar unos contenidos de género determinados? Si es así, ¿̇en relación a qué ámbitos disciplinares y/o cronológicos? ¿Qué temáticas o esferas prefieren? 
- ¿ ¿Consideran que la introducción de la historia de las mujeres o la perspectiva de género en la enseñanza podría contribuir de alguna forma a la transformación de las estructuras sociales que promueven la discriminación por razones de género y la desigualdad?

Estas preguntas están igualmente relacionadas con los objetivos, entendidos como las metas hacia las que se encamina la investigación y que, a su vez, están conectados con la finalidad o finalidades de la misma. En nuestro caso concreto, podemos identificar un total de cinco objetivos que enunciamos a continuación:

- Conocer el grado de formación en género de los estudiantes del Máster Universitario de Formación en Profesor/Profesora de Enseñanza Secundaria (MAES) de la Universidad de Valencia, mayoritariamente egresados de la facultad de historia.

- Entender su valoración de las aportaciones realizadas por la historia de las mujeres y por la investigación y la docencia desde la perspectiva de género a partir de su experiencia formativa.

- Comparar y valorar la utilidad que le asignan a la inclusión de las mujeres en la enseñanza. Contrastar y comprender las elecciones de protagonistas que realizan y la utilidad que les asignan.

- Identificar y analizar sus propuestas para introducir la enseñanza de la historia de las mujeres en las aulas. Interpretar y contrastar el discurso del profesorado sobre la inclusión de las mujeres y su historia con las prácticas que realizan.

- Valorar cómo entienden el cambio que esto puede introducir en las aulas y en la sociedad en general.

Aunque por razones de espacio no podemos incluir todos los elementos apuntados, presentaremos a continuación, en al apartado de resultados, algunos elementos relacionados con dos de las variables de análisis consideradas: 1) el grado de formación inicial en género de los futuros docentes de historia y las formas que esta ha adoptado (transversal o específica); 2) la valoración que dichos estudiantes realizan de las aportaciones de la perspectiva de género a la enseñanza y el aprendizaje de la historia, la geografía o la historia del arte.

Con el fin de realizar el análisis de los datos recabados hemos establecido tres niveles en función de la formación en género recibida por los participantes: a) estudiantes que han cursado una asignatura específica de género; b) estudiantes que refieren haber recibido formación de género de forma transversal o como parte de alguna otra asignatura; c) estudiantes sin formación de género. En un segundo nivel, hemos procedido a analizar la pregunta del cuestionario que interroga a los participantes acerca de su valoración de la perspectiva de género. Para ello hemos establecido una serie de unidades de significado o categorías temáticas que hemos nombrado a partir de la asignación de un código (Creswell 2014; Miles y Huberman, 1994; Simons, 2011 ; Stake, 2007). Dichas codificaciones han sido, posteriormente, agrupadas en tres grandes categorías: a) las mujeres como sujetos en la historia; b) el género como enfoque o perspectiva que cuestiona los planteamientos de la historia tradicional y su carácter androcéntrico; c) el género en su dimensión social, como un instrumento que permite describir mejor 
o transformar la realidad. Hemos añadido una cuarta categoría, una especie de cajón de sastre, etiquetada como "otros", donde incluimos aquellas codificaciones que aparecen con una recurrencia menor y que no están relacionadas con ninguna de las anteriores (identidades, sexo y diferencia biológica, roles de género, aproximación binaria femenino-masculino...).

A modo de ejemplo, transcribimos algunas de las respuestas debidamente anonimizadas. El indicador inicial $(H \circ M)$ identifica el género señalado por los y las participantes. El número que le sigue ha sido asignado a los cuestionarios de forma aleatoria. Finalmente, la última letra alude a la formación recibida en materia de género: específica $(E)$, transversal $(T) \circ \sin$ formación $(S){ }^{3}$

- La teoría de género, junto a los estudios culturales o coloniales son una parte muy importante de la historiografía, pero considero que -igual como antes con el marxismo-, basar toda explicación en clave de género puede llegar a ser reduccionista (H6T).

- La perspectiva de género completa la historia de alguna manera (M18S).

- Es imprescindible enseñarla, porque las mujeres somos parte de la historia (M21T).

- Las mujeres han sido también sujetos de la historia y han participado en su construcción (M26E).

- (El género) es rescribir la historia desde la perspectiva de las mujeres (H7S).

- El enfoque de género da voz a un sector democrático tradicionalmente invisibilizado, el de las mujeres o identidades de género tampoco escuchadas. Es revolucionario e implica una revisión total del discurso histórico dominante (H21E).

- Siempre ha estado ausente y considero que esto significa eliminar una buena parte de la historia (H37E).

- El papel de la mujer siempre ha estado relegado a un segundo plano en el marco de las ciencias sociales. Es necesario sacar su papel a la luz (M9T).

- Somos más relevantes de lo que creen (M12T).

- El mundo está socialmente construido desde la perspectiva de la figura masculina y a ojos de ellos (mayoritariamente) y por tanto no es real o no se ajusta correctamente a la realidad (M13T).

- Una disciplina que tiene por objeto la sociedad no puede omitir al 50\% de la misma. Sin mujer no hay historia, no hay arte, no hay geografía (M25E).

3 A modo de ejemplo, las siglas H6T vendían a significar: hombre, cuestionario $n^{\circ} 6$, con formación transversal en género. Las cuatro primeras respuestas transcritas corresponden a cuestionarios del curso 2016-2017; las siete restantes, al curso 2017-2018. 


\subsection{Los datos de la muestra}

Como señalábamos, la muestra está constituida por 70 alumnos del Máster Universitario de Formación en Profesor/Profesora de Enseñanza Secundaria (MAES) de la Universidad de Valencia, que cursaban la asignatura de "Complementos para la formación disciplinar» durante los cursos 2016-2017 y 2017-2018. Se trata mayoritariamente de egresados procedentes de los grados de Historia, Historia del Arte y Geografía y Medio Ambiente, con un marcado predominio del grado de Historia (59,98\%) frente a los otros dos, Historia del Arte $(21,42 \%)$ y Geografía y Medioambiente (10\%). Como indican las tablas que incluimos a continuación, un porcentaje pequeño de los estudiantes procede de otras titulaciones: Humanidades, Sociología y Periodismo. Por lo que respecta al perfil de género, documentamos un ligero predominio de los estudiantes varones $(54,26 \%)$ frente a las mujeres $(45,69 \%)$, lo que corrobora lo apuntado por la bibliografía existente, que subraya cierta tendencia a la masculinización en grados como el de historia (Bolufer, 2018). La edad media de los participantes se sitúa ligeramente por encima de los 24 años.

\section{Tabla 1}

Datos de la muestra: $n .^{\circ}$ de estudiantes, titulación, curso académico, estudios de procedencia

\begin{tabular}{|c|c|c|c|}
\hline $\mathrm{N}^{\circ}$ & Titulación & Curso & Estudios de procedencia \\
\hline 33 & $\begin{array}{l}\text { Máster universitario de } \\
\text { profesor/a de secundaria }\end{array}$ & $2016-2017$ & $\begin{array}{l}\text { Grados de H. }{ }^{a} \text { del Arte, Historia, } \\
\text { Geografía y Humanidades. }\end{array}$ \\
\hline 37 & $\begin{array}{l}\text { Máster universitario de } \\
\text { profesor/a de secundaria }\end{array}$ & $2017-2018$ & $\begin{array}{l}\text { Grados de } \mathrm{H}^{\text {a }} \text { del Arte, Historia, } \\
\text { Geografía, Humanidades, } \\
\text { Sociología y Periodismo. }\end{array}$ \\
\hline
\end{tabular}

Fuente: elaboración propia.

Tabla 2

Estudios de origen de los estudiantes que integran la muestra

\begin{tabular}{lcccccc}
\hline & Historia & $\mathrm{H}^{{ }^{a}}$ del Arte & Geografía & Otros & $\mathrm{NC}$ & Total \\
\hline Curso 2016-2017 & 21 & 6 & 5 & 1 & 0 & 33 \\
Curso 2017-2018 & 21 & 9 & 2 & 3 & 2 & 37 \\
\hline TOTAL & 42 & 15 & 7 & 4 & 2 & 70 \\
\hline$\%$ & $59^{\prime} 98 \%$ & $21,42 \%$ & $10 \%$ & $5^{\prime} 72 \%$ & $2{ }^{\prime} 86 \%$ & $100 \%$ \\
\hline
\end{tabular}

Fuente: elaboración propia.

Tabla 3

Perfil de género de la muestra: número de hombres y mujeres por curso académico y porcentaje sobre el global

\begin{tabular}{lcccc}
\hline Curso académico & $2016-2017$ & $2017-2018$ & Total & $\%$ \\
\hline N..$^{\circ}$ de hombres & 19 & 19 & 38 & $54^{\prime} 26 \%$ \\
N. ${ }^{\circ}$ de mujeres & 14 & 18 & 32 & $45^{\circ} 69 \%$ \\
\hline Total & 33 & 37 & 70 & $100 \%$ \\
\hline
\end{tabular}

Fuente: elaboración propia. 


\section{Resultados y discusión}

\subsection{La formación en género de los futuros docentes de historia}

Uno de los primeros elementos que quisiéramos destacar es el alto número de participantes que refieren haber recibido algún tipo de formación en género. Más de la mitad (56,5\%) han recibido formación específica, esto es, han cursado al menos una asignatura consagrada exclusivamente a género. Este porcentaje se eleva hasta el $76,85 \%$ de la muestra si consideramos aquellos que, pese a no haber cursado ninguna asignatura de género en grado o posgrado, sí han abordado cuestiones de género de forma transversal. Pese a ello, el número de aquellos que nunca se han encontrado con el género a lo largo de su formación inicial es algo superior $(23,2 \%)$ al de quienes que refieren haberlo estudiado de forma transversal en alguna de las asignaturas que han integrado su formación de grado y/o posgrado (20,3\%) (tabla 4). Las titulaciones de aquellos que refieren no haber recibido ningún tipo de instrucción son: historia del arte, geografía e historia, historia, geografía y medioambiente, periodismo y humanidades.

Tabla 4

Formación en género de los participantes

\begin{tabular}{llc}
\hline & N. & \multicolumn{1}{c}{$\%$} \\
\hline Estudiantes que han cursado una asignatura específica de género & 39 & $56^{\prime} 5 \%$ \\
$\begin{array}{l}\text { Estudiantes que refieren haber recibido formación de género de forma } \\
\text { transversal o como parte de alguna otra materia }\end{array}$ & 14 & $20{ }^{\prime} 3 \%$ \\
Estudiantes sin formación de género & 16 & $23{ }^{\prime} 2 \%$ \\
Total & 69 & 100 \\
\hline
\end{tabular}

Fuente: elaboración propia.

Los alumnos que manifiestan haber cursado materias específicas de género han recibido dicha formación muy mayoritariamente en sus estudios de grado, concretamente en el grado de historia. Un porcentaje más reducido refiere haberse matriculado en alguna materia de género como parte de su formación de postgrado $(5,8 \%)$, en másteres propios o ajenos a la Universidad de Valencia. Con ello, es la asignatura "Mujeres y hombres en la historia" la que parece marcar la diferencia, ya que ha sido seguida por algo más de la mitad de los participantes en la encuesta $(50,7 \%)$, como parte de sus estudios del grado de historia. Vigente desde el curso 2013-2017, es esta una asignatura de carácter obligatorio de seis créditos que se imparte en el primer semestre del último año de la titulación. Es, igualmente, una materia que goza de una trayectoria de más de veinte años en la universidad, como continuadora de otras dos que le precedieron: "Hombres y mujeres en la sociedad y la cultura occidental", de tercer curso que fue introducida en el Plan de estudios de 1994 de la Licenciatura en Historia (cuatrimestral y de 4,5 créditos); "Hombres y mujeres en la Historia", en las áreas de Historia Antigua, Historia Moderna e Historia Contemporánea (optativa anual) (Bolufer, 2018).

A nuestro juicio, resulta también relevante el número de participantes que refiere haber recibido formación en género de manera transversal en materias generalistas o como parte del temario de otras asignaturas. Se trata, mayoritariamente, de alumnos procedentes del grado de arte, pero también del de historia. Hemos encontrado menciones a un total de once materias: Historia 
de la fotografía, Historia del Cine y otros medios audiovisuales I y II, Arte actual, Historia de las artes decorativas europeas, Historia de las Ideas Estéticas (grado de Historia del Arte); Historia Antigua Universal I y II, Metodología e Historiografía, Historia del Arte I y II (grado de Historia).

\subsection{Las valoraciones sobre la perspectiva de género y sus aportaciones a la docencia y a la investigación. Las codificaciones}

Consideramos en este apartado la valoración que los participantes realizan de las aportaciones de la perspectiva de género a la enseñanza y el aprendizaje de la historia y/o las restantes ciencias sociales. Sus respuestas, entendidas de forma binaria (un escueto sí o no), apenas aportan información. En la pregunta: żconsideras relevantes las aportaciones de la perspectiva de género a la historia y a las restantes ciencias sociales?, tan solo documentamos un ítem en blanco para el curso $2016-2017$, lo que nos proporciona un abrumador $98,55 \%$ de respuestas afirmativas. Sin embargo, creemos que debemos tomar esta cifra con cierta cautela porque cuando, más adelante, los estudiantes deban concretar ese supuesto interés explicitando cuáles han sido las aportaciones de la perspectiva de género a la historia, el número de respuestas en blanco o de aquellas en las que estos se muestran incapaces de contestar se multiplica por diez, hasta alcanzar el $14,4 \%$ del total.

En el análisis de las respuestas hemos operado en dos niveles: 1) el establecimiento de una serie unidades de significado o categorías temáticas que han sido identificadas a partir de la asignación de un código (codificaciones); 2) el agrupamiento de todas ellas en una serie de unidades mayores (categorías). En un primer momento, hemos podido identificar hasta 19 codificaciones diferentes, que aparecen numeradas y ordenadas por frecuencia decreciente en la tabla 5. Algunas de ellas se documentan con una presencia casi residual, ya que constan en tan solo una o dos ocasiones. Es el caso de la consideración o valor diferencial de las aportaciones de hombres y mujeres; las alusiones a los comportamientos y roles de género; el género como categoría de análisis y su relación con la historiografía; su actualidad y relevancia social; su definición como construcción social y cultural de la diferencia sexual o las alusiones al sexo biológico o la diferencia sexual (Beauvoir, 2005; Butler, 1999 y 2006; Scott, 1990). En sentido contrario, un puñado de codificaciones se reiteran una y otra vez en las respuestas de los participantes. Se trata, especialmente, de las menciones al silencio, la invisibilidad $\circ$ la marginación de las mujeres (recurrencia de 41 ) o de aquellas que se refieren al género como perspectiva, punto de atención, punto de vista o enfoque (recurrencia de 30). En una horquilla bastante menor (recurrencia de 13 a 15), aparecen otras codificaciones que destacan la importancia de la perspectiva de género a la hora poner en valor las aportaciones, los logros o el papel de las mujeres en la historia, la denuncia de la prevalencia del androcentrismo en el relato histórico tradicional o la constatación de que la inclusión del sesgo de género implica una aproximación más fiel a la realidad social y contribuir a transformarla. En esta misma línea, queremos destacar la relación entre género y equidad, la no discriminación, las alusiones al feminismo $\circ$ aquellas que vinculan género e identidades (al referirse a identidad individual $\circ$ social, identidades de género, auto-concepto o formas de sentirse hombre o mujer). Por último y con una frecuencia muy baja ( $3 \circ 4$ referencias), constan algunas alusiones al patriarcalismo, a la forma en la que el género contribuye a la formación de ciudadanos o al desarrollo del pensamiento crítico, pero también la aproximación relacional marcada por el binomio masculino y femenino como visión significante del mundo (Bourdieu, 1997) o una concepción más clásica que rescata para la historia tan solo a las mujeres importantes (Figura 1). 


\section{Tabla 5}

Recurrencia de las codificaciones empleadas para valorar el interés de la perspectiva de género

\begin{tabular}{lc}
\hline \multicolumn{1}{c}{ Codificación } & Recurrencia \\
\hline 1. Mujeres invisibilizadas, olvidadas, marginadas & 41 \\
2. Perspectiva (punto de atención, punto de vista, enfoque) & 30 \\
3. Aportaciones, logros, papel (de las mujeres) & 15 \\
4. Sociedad o realidad (conocer y/o cambiar) & 14 \\
5. Androcentrismo (del relato histórico, hombres importantes...) & 13 \\
6. Equidad, igualdad & 7 \\
7. En oposición a la historia tradicional & 7 \\
8. Identidades & 6 \\
9. Feminismo & 5 \\
10. Mujeres importantes & 4 \\
11. Formación de ciudadanos / Desarrollo del pensamiento crítico & 4 \\
12. Patriarcalismo & 3 \\
13. Aproximación relacional (hombres y mujeres; masculino y femenino) & 3 \\
14. Comportamientos y roles de género & 2 \\
15. Género como construcción social y cultural & 2 \\
16. Sexo (biológico, orientación sexual, diferencia sexual) & 2 \\
17. Valor diferencial de las aportaciones de hombres y mujeres & 1 \\
18. Aportaciones del género a la historiografía & 1 \\
19. Actualidad, relevancia social & 1 \\
\hline
\end{tabular}

Fuente: elaboración propia.

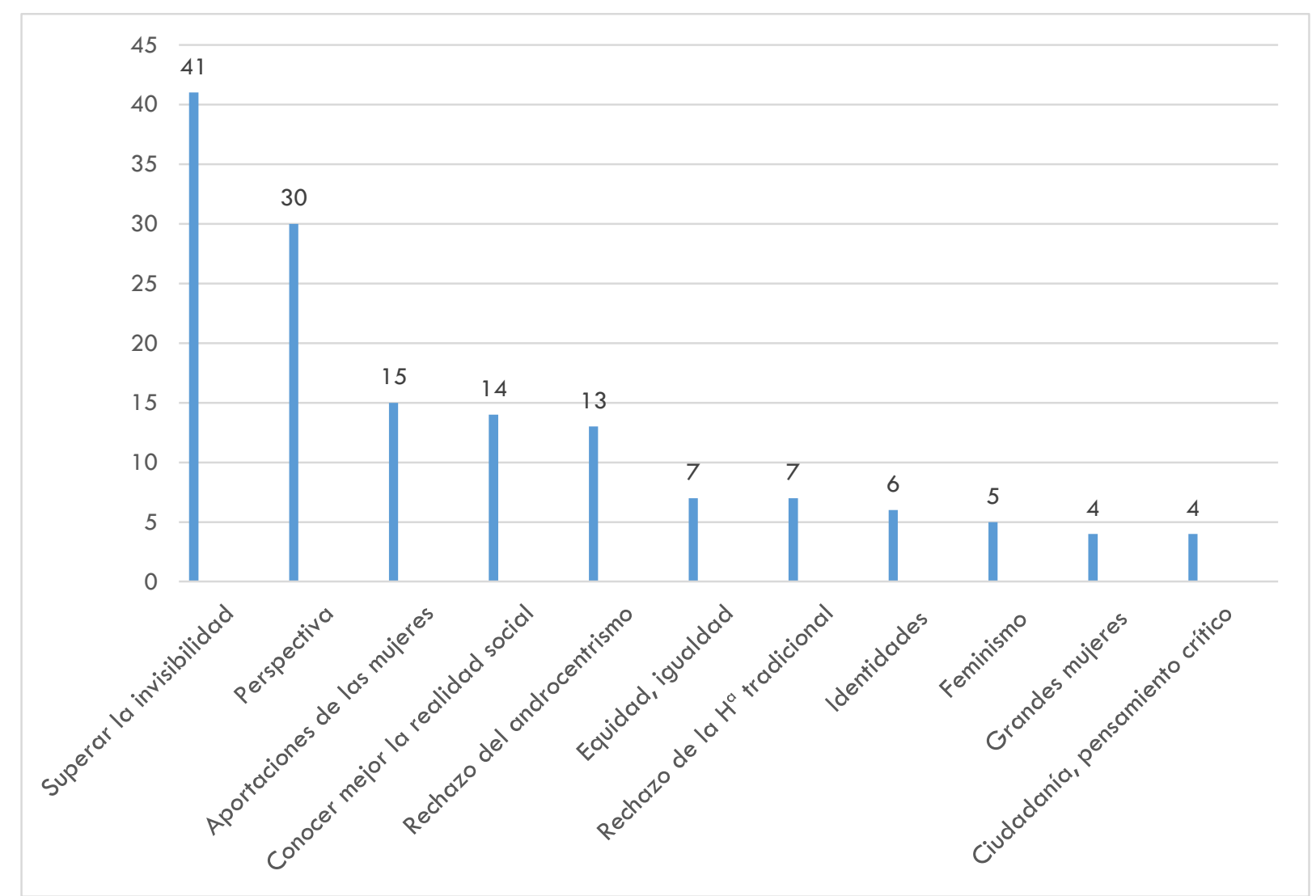

Figura 1. Recurrencia de las codificaciones más utilizadas por nuestros estudiantes para valorar el interés de la perspectiva de género. Fuente: elaboración propia. 


\subsection{El establecimiento de las categorías}

Otro nivel de reducción para realizar el agrupamiento de los datos brutos del registro es aquel que los organiza en categorías temáticas, de modo que las unidades que emergen permiten que el investigador contraste, compare, analice y ofrezca modelos de interpretación $y / 0$ de comprensión (Álvarez-Gayou, 2003; Creswell, 2014; Miles y Huberman, 1994; Wolcott, 1994; Simons, 2011 ; Stake, 2007). Ello nos permite agrupar las codificaciones expuestas en las cuatro categorías referidas en el apartado de metodología: a) las mujeres como sujetos de la historia; b) el género como enfoque o perspectiva; c) el género en su dimensión social; d) otras.

\section{Tabla 6}

Categorización establecida a partir de las codificaciones

\begin{tabular}{ll}
\hline \multicolumn{1}{c}{ Categorías } & \multicolumn{1}{c}{ Codificación } \\
\hline \multirow{2}{*}{ de la hujeres como sujetos } & A.2 Valor diferencial de hombres y mujeres \\
& A.3 Mujeres importantes \\
& A.4 Mujeres invisibilizadas, marginadas... \\
El género como categoría & B.1. Aportaciones del género a la historiografía \\
de análisis, perspectiva o & B.2. Perspectiva (punto de atención o de vista, enfoque) \\
enfoque & B.3. En oposición a la historia tradicional \\
& B.4 Androcentrismo (del relato, grandes hombres) \\
& C.1. Equidad, igualdad, respeto, discriminación \\
Feminismo y cambio social & C.2. Feminismo \\
& C.3. Patriarcalismo \\
& C.4. Actualidad, relevancia social. \\
& C.5. Sociedad o realidad (conocer y/o cambiar) \\
& C.6. Formación ciudadana / Pensamiento crítico \\
& D.1. Aproximación relacional (hombres y mujeres; \\
masculino y femenino) \\
D.2. Comportamientos y roles de género \\
D.3. Género como construcción social y cultural \\
D.4. Sexo (biológico, orientación, diferencia sexual) \\
D.5. Identidades
\end{tabular}

Fuente: elaboración propia.

\section{Tabla 7}

Número de codificaciones por categoría y peso relativo de cada categoría en la valoración de la perspectiva de género

\begin{tabular}{llllll}
\hline Categoría & $\mathrm{A}$ & $\mathrm{B}$ & $\mathrm{C}$ & $\mathrm{D}$ & Total \\
\hline Recurrencia & 61 & 51 & 34 & 15 & 161 \\
$\%$ & $37^{\prime} 8$ & $31^{\prime} 62$ & $21^{\prime} 08$ & $9^{\prime} 5$ & 100 \\
\hline
\end{tabular}

Fuente: elaboración propia.

La primera de las categorías es también aquella que goza de un mayor peso porcentual sobre el total (37,8\%) (Figura 2). Con ello, nuestros alumnos recogen en primer lugar la que ha sido una de las grandes aportaciones de la historia de las mujeres: su recuperación como sujetos históricos (Fernández, 2001; Scott, 1990). La segunda categoría identifica el género como perspectiva o 
enfoque o como instrumento de análisis, además de valorar sus aportaciones historiográficas. Posee un peso que es, porcentualmente, solo algo inferior a la anterior $(31,62 \%)$ y aparece bajo la forma de respuestas que denuncian la prevalencia del androcentrismo en el relato de las ciencias sociales o el rechazo a otras formas más tradicionales de entender la historia (Crocco, 1997; Lerner, 1979; Woyshner, 2002). Sin embargo, creemos necesario indicar que la presencia relativa de dicha categoría aumenta porque casi la mitad de los estudiantes de la muestra repiten en su respuesta la palabra "perspectiva", que estaba presente en el enunciado de la cuestión. Si eliminásemos estas respuestas (correspondientes a la codificación B.2), el peso porcentual de la segunda categoría se reduciría a tan solo el 13,02\% del total. Finalmente, la categoría denominada "feminismo y cambio social" refiere la lucha por la igualdad, pero también la forma en la que la inclusión de la perspectiva de género permite una aproximación más veraz a la realidad de las sociedades presentes y pasadas. Su peso relativo es también importante $(21,08 \%)$, aunque aquí las respuestas tienden a parecer algo más fragmentadas. Destacan especialmente las que subrayan cómo formar o formarse en género significa conocer mejor la sociedad o contribuir a cambiarla, el modo en que el género contribuye a una mayor justicia social o a la igualdad y su vinculación con el movimiento feminista.

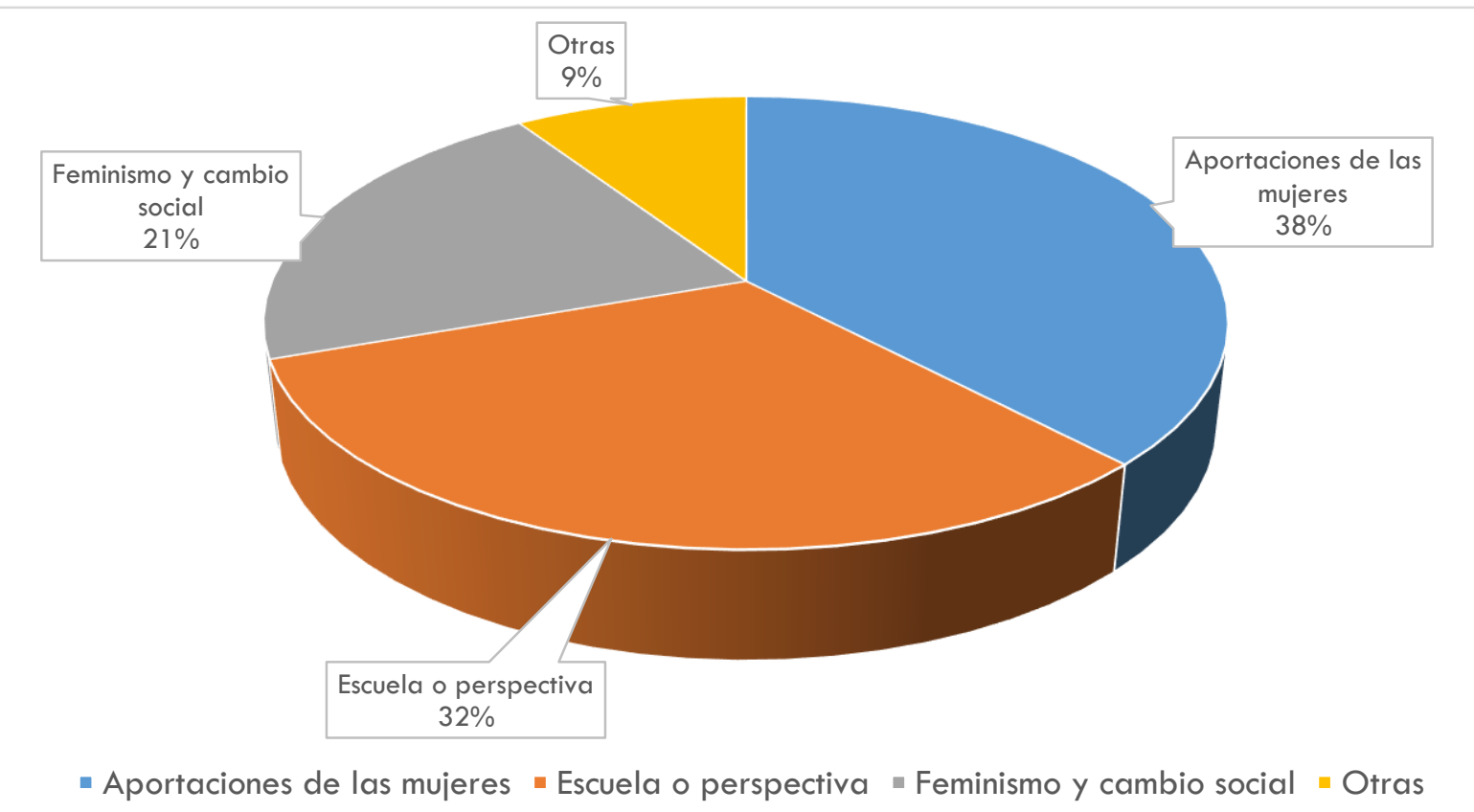

Figura 2. Peso porcentual de las distintas categorías referentes a la valoración del interés de la perspectiva de género. Fuente: elaboración propia.

\subsection{Las variaciones según la formación recibida y el género de los encuestados}

En este último apartado quisiéramos profundizar en los resultados expuestos, incluyendo otros dos elementos de análisis: 1) la formación previa en género de los participantes; 2) la identidad de género señalada como propia al inicio del cuestionario.

La formación recibida en género parece introducir una primera variante: los estudiantes sin formación tienden a plantear respuestas más sencillas, con solo 1-2 codificaciones diferentes por respuesta. Sin embargo, la complejidad de las aportaciones de los participantes aumenta con el nivel de formación, ya que aquellos que han recibido algún tipo de formación en género tienden 
a incluir un número relativamente superior de codificaciones (entre 2 y 3 ), sin que haya mucha diferencia en función de si dicha formación ha sido específica o transversal. Observamos igualmente una diferencia en la tipología de las categorías que se incluyen en las respuestas: los estudiantes sin formación activan, preferentemente, la categoría A (aportaciones de las mujeres), mientras que los estudiantes con formación prefieren las categorías B y C (perspectiva o enfoque; feminismo y cambio social). Respecto a las codificaciones, el primer subgrupo tiende activar aquellas que refieren la invisibilidad de las mujeres para la historia o las referencias al binomio hombre - mujer. Tiende, igualmente, a repetir en sus respuestas el término perspectiva. Por el contrario, un mayor nivel de formación, transversal o específica, implica una mayor recurrencia de las codificaciones relacionadas con la oposición a la historia tradicional, el post-estructuralismo feminista, el sexo y la diferencia biológica o las identidades.

Por lo que respecta a las variaciones en función del género de los participantes, queremos en primer lugar recordar que todos ellos se identificaron recurriendo al binomio hombre - mujer, sin optar por otras posibilidades. Esta identificación parece introducir también un sesgo en los resultados, matiz que se evidencia en la categorización y que se muestra de forma aún más marcada en la elección de algunas de las codificaciones. Las categorías A y B -esto es, aquellas que se refieren a las mujeres como sujetos de la historia y el género como perspectiva, corriente - enfoque- son elegidas en términos de casi igualdad tanto en el subgrupo de las mujeres como en el de hombres (un $57 \%$ del total de las encuestadas y de los encuestados opta por la categoría $A$, mientras que en el caso de la categoría B las cifras oscilan entre el $31 \%$ de los varones y el $35 \%$ de las mujeres). Sin embargo, en la categoría $C$, correspondiente a feminismo y cambio social, los participantes masculinos duplican las alusiones al feminismo, el patriarcalismo $\circ$ a las desigualdades (peso relativo del $36^{\prime} 82 \%$ en los varones, frente a solo el $14,28 \%$ entre las mujeres).

En lo tocante a las codificaciones, ellos son también más proclives a sustentar el interés de la perspectiva de género en aquella definición que lo entiende como la construcción social y cultural de la diferencia sexual (el 15,7\%, frente a ninguna mujer). Los varones recurren con mayor frecuencia a la aproximación relacional (binario hombre-mujer), que está presente en un $47,4 \%$ de los encuestados masculinos frente al $35 \%$ de las féminas. Finalmente, son ellos igualmente quienes recurren más a las codificaciones relativas a las identidades, individuales o sociales, identidades de género, queer o LGTBI+, una codificación que aparece en el $21 \%$ de las respuestas masculinas frente a solo el $7^{\prime} 14 \%$ de las femeninas. En sentido contrario, las mujeres son cuatro veces más proclives que los hombres a subrayar el androcentrismo del relato tradicional de la historia (presente en el $42 \%$ de las respuestas femeninas) frente a la aparente ceguera de sus compañeros varones, quienes solo consignan esta codificación en el 5,26\% de los casos. En una línea similar, la mitad de las respuestas femeninas denuncian la invisibilidad de las mujeres para las ciencias sociales o la forma en la que ellas son parte de los denominados "olvidos de la historia" (49,98\%), un elemento que tan solo está presente, en cambio, en una de cada seis respuestas masculinas (15,78\%). Igualmente, el $42,8 \%$ de las participantes alude a la forma en la que el género subraya las aportaciones, los logros y el papel de las mujeres en la historia, algo que señalan solo el $26,3 \%$ de los encuestados. Son ellas, finalmente, las únicas que arguyen que estudiar el género proporciona una visión más ajustada de la realidad social, contribuyendo a cambiarla $(21,42 \%)$ (Figura 3). 


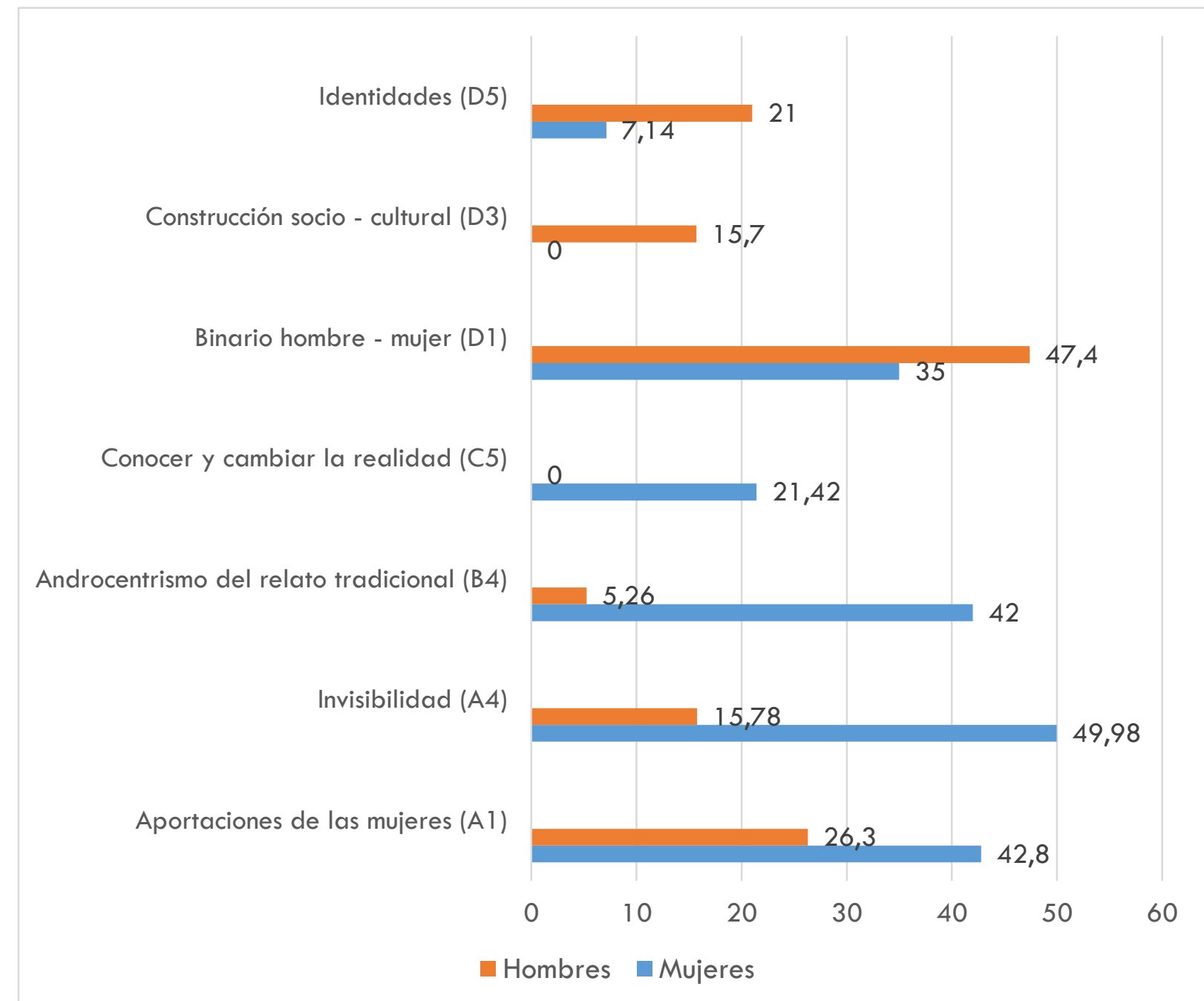

Figura 3. Sesgo de género: variación de las codificaciones en función del género del encuestado (el valor se indica en \% sobre el total de las respuestas femeninas y masculinas). Fuente: elaboración propia.

\section{Reflexiones finales}

El objetivo de nuestro trabajo era, como señalábamos al inicio, relacionar los conceptos de género y formación de docentes de historia para conocer mejor el valor que los docentes en formación otorgan a las aportaciones realizadas por la perspectiva de género. A grandes rasgos, los participantes consideran que la perspectiva de género contribuye, especialmente, a poner fin a la invisibilidad, el olvido y la marginación de las mujeres en la historia, recuperando sus aportaciones y sus logros. En un segundo nivel, conectan género y realidad social, relacionándolos con una mayor equidad y con el feminismo, para finalmente valorar, aunque con un peso relativo menor, la forma en la que el género critica el discurso tradicional de la historia, especialmente en relación al carácter androcéntrico de dicho relato.

Sin embargo, la introducción del género como elemento de análisis dentro de la propia investigación nos obliga a matizar los resultados expuestos. Los varones parecen replicar en mayor modo el binomio hombre - mujer / masculino - femenino, al tiempo que son ellos también quienes subrayan más la relación entre género e identidades. Quizá no sorpresivamente son ellas, en cambio, quienes muestran una mayor sensibilidad hacia la ausencia femenina del relato de la historia, la prevalencia del varón, la forma en la que el género recupera para el presente las 
voces de las mujeres del pasado o cómo permite describir en términos diferentes la realidad social. Todo ello parece sugerir una recepción dispar de las aportaciones de la perspectiva de género entre los y las futuras docentes de historia, sugiriendo nuevas líneas de investigación y apuntando la posible necesidad de diseñar e implementar estrategias específicas de trabajo en el aula que tengan en consideración el género de los estudiantes.

Si consideramos el género un elemento básico para el desarrollo, este no puede seguir siendo, únicamente, "cosa de mujeres". Una sociedad basada en la igualdad de género es también una sociedad más justa $y$, consecuentemente, una sociedad mejor para todos. Como señala Schiebinger (2018):

Al menos ya hemos abierto los ojos y no podemos volver a un mundo que ignora el género. La innovación es lo que mueve al mundo. Espero haber empezado ya a demostrar que las innovaciones de género despiertan la creatividad ofreciendo nuevas perspectivas, planteando nuevas preguntas y abriendo nuevas áreas de investigación ¿̇Podemos permitirnos seguir ignorando estas oportunidades? (p. 35).

\section{Agradecimientos}

Este trabajo ha sido realizado dentro del proyecto "Las representaciones sociales de los contenidos escolares en el desarrollo de las competencias docentes" del Ministerio de Ciencia, Innovación y Universidades (PGC2018-094491-B-C32).

\section{Bibliografía}

Alaminos, A. y Castejón, J. L. (2006). Elaboración, análisis e interpretación de encuestas, cuestionarios y escalas de opinión. Alicante: Universidad de Alicante.

Alario, T. (2008). Arte y Feminismo: Your Body Is a Battleground. Nerea: San Sebastián.

Alonso, A. M. (1998). El sexismo en el aula. Los valores y la didáctica de las Ciencias Sociales. Actas del IX Simposium de Didáctica de las Ciencias Sociales (pp. 309-316). Lleida: Universitat de Lleida.

Álvarez-Gayou, J. L. (2003). Cómo hacer investigación cualitativa. Fundamentos y metodología. México: Paidós.

Aresti, N. (2010). Masculinidades en tela de juicio. Madrid: Cátedra.

Beauvoir, S. (2005). El segundo sexo. Madrid: Cátedra (1. a edición de 1949).

Bereni, L., Chauvin, S., Jaunait, A. y Revillard, A. (2008). Introduction aux "gender studies"). Manuel des études sur le genre. Bruxelles: De Boek.

Blanco, N. (2000). El sexismo en los materiales educativos de la ESO. Sevilla: Instituto andaluz de la mujer.

Bolufer, M. (2018). Mujeres y hombres en la historia. Una propuesta historiográfica y docente. Granada: Comares.

Bourdieu, P. (1997). Masculine Domination Revisited. Berkeley Journal of Sociology. Youth and youth culture, 41, 189-203. 
Butler, J. (1999). El género en disputa. El feminismo y la subversión de la identidad. México: Paidós.

Butler, J. (2006). Deshacer el género. Barcelona: Paidós lbérica.

Creswell, J. W. (2014). Research design. Qualitative, quantitative and mixed methods approaches. California: Sage.

Crocco, M. S. (1997). Making time for women's history. When your survey course is already filled to overflowing. Social Education, 61(1), 32-37.

Crocco, M. S. (2006). Gender and social education: What's the problem? En Ross, E. W. (ed.), The social studies curriculum: Purposes, problems, and possibilities, (pp. 171-193). Albany: State University of New York Press.

Crocco, M. S. y Cramer, J. (2005). Technology use, women, and global studies in social studies teacher education. Contemporary Issues in Technology and Teacher Education, 5(1), 38-49.

Crocco, M. S. y Libresco, A. S. (2007). Gender and social studies teacher education. En S. Sadler y E. Silber (eds.), Gender and teacher education: exploring essential equity questions (pp. 109164). Mahwah, NJ: Erlbaum.

De Lauretis, T. (1989). Technologies of gender. Essays on theory, film and fiction. Londres: Mcmillan Press.

Eichler, M. (1991). Non-sexist research methods. A practical guide. Nueva York - Londres: Routledge.

Fernández, A. (2001). Las mujeres en la enseñanza de las Ciencias Sociales. Madrid: Síntesis.

Foucault, M. (1990). Vigilar y castigar: nacimiento de la prisión. Madrid: Siglo XXI.

Foucault, M. (2005). La inquietud de sí. Madrid: Siglo XXI.

Garreta, N. y Careaga, P. (1987). Modelos masculino y femenino en los textos E.G.B. Madrid: Serie Estudios 14.

Harding, S. (1987). Feminism and methodology. Bloomington: Indiana University Press.

Hidalgo, E., Juliano, D., Roset, M. y Caba, A. (2003). Repensar la enseñanza de la geografía y la historia. Una mirada desde el género. Barcelona: Octaedro.

Hustvedt, S. (2017). La mujer que mira a los hombres que miran a las mujeres. Ensayos sobre feminismo arte y ciencia. Barcelona: Seix Barral.

Lather, P. (1991). Getting smart: feminist research and pedagogy with / in the post-modern. Nueva York: Routledge.

Lerner, G. (1979). The majority finds its past: placing women in history. Oxford - Nueva York: Oxford University Press.

Lerner, G. (1993). The creation of feminist consciousness. Oxford - Nueva York: Oxford University Press.

López-F. Cao, M. (2001). Modelos, conductas y estereotipos femeninos en la creación icónica. En A. Fernández. Las mujeres en la enseñanza de las Ciencias Sociales (pp. 169-205). Madrid: Síntesis. 
López-Navajas, A. (2014). Análisis de la ausencia de las mujeres en los manuales de la ESO: una genealogía de conocimientos ocultada. Revista de educación, 363, 282-308.

Mclntosh, P. (1983). Interactive phases of curricular re-vision. Working Paper, 124.

Miles M. B. y Huberman, A. M. (1994). Qualitative data analysis: a sourcebook of new methods. Thousand Oaks, CA: Sage.

Minnich, E. K. (1990). Transforming Knowledge. Philadelphia: Temple University Press.

Mouffe, Ch. (1993). Feminismo, ciudadanía y política democrática radical. Debate feminista, 4(7), 3-22.

Morant, I., Segura, C., Di Febo, G. y Perry, M. ${ }^{a}$ E. (2013). Arenal y la historiografía feminista española e hispanista en las dos últimas décadas. Arenal, 20(1), 81-105

Morant, I. (2017). Mujeres e historia. La construcción de una historiografía. En Baena, A. y Roselló, E. (coord.), Históricas digital. Mujeres en la Nueva España (pp. 25-54). México: Universidad Nacional Autónoma de México - Instituto de Investigaciones Históricas. Recuperado de http://www.historicas.unam.mx/publicaciones/publicadigital/libros/mujeres/673 0403 Isabel Morant.pdf

Nochlin, L. (1989). Women, Art, and Power and other Essays. Londres - Nueva York: Taylor \& Francis.

Oakley, A. (1998). Gender, methodology and people's ways of knowing: some problems with feminism and the paradigm debate in social science. Sociology, 32(4), 707-731.

Opériol, V. (2013). Le genre en histoire: la construction du féminin et du masculin. Le cartable de Clio, 13, 15-23.

Pagés, J. y Sant, E. (2012). Las mujeres en la enseñanza de la historia: ¿Hasta cuándo serán invisibles? Cadernos de Pesquisa do Cdhis, 25(1), 91-117.

Rausell, H. y Talavera, M. (2019). Género y didácticas. Una mirada crítica, una aproximación práctica. Valencia: Tirant lo Blanch.

Rausell, H. (2019). La historia de las mujeres y el derecho al pasado. En Parra, D. y Fuertes, C. (eds.), Reinterpretar la tradición, transformar las prácticas (pp. 237-253). Valencia: Tirant lo Blanch.

Rose, S. O. (2010) ¿Qué es historia de género? Madrid: Alianza Editorial.

Rugna, C. M. (2014). Feministas en sus zapatos: historiografía y enseñanza de la historia. Clio \& Asociados. La enseñanza de la historia, 18, 216-231.

Ruiz, A. (2009). Método de encuesta: construcción de cuestionarios, pautas y sugerencias. Reire. Revista d'Innovació i Recerca en Educació, 96-110.

Schiebinger, L. (2004). ¿̇Tiene sexo la mente? Madrid: Cátedra.

Schiebinger, L. (ed.) (2008). Gendered innovations in science and engineering. Standford: Standford University Press.

Schiebinger, L. (2018). Discurs d'investidura com a doctora "Honoris Causa" per la Universitat de València. Valencia: Universidad de Valencia. 
Schneuwly, B. (2015). La didactique des disciplines peut-elle intégrer les questions de genre? Genre, didactique, formation (pp. 2-6). París: Centre Hubertine Auclert.

Scott, J. W. (1990). El género, una categoría útil para el análisis histórico. En J. Amelang y M. Nash (comps.). Historia y género. Las mujeres en la Europa moderna y contemporánea (pp. 23-56). Valencia: Alfons el Magnànim.

Scott, J. W. (2008). Género e Historia. México: FCE.

Simons, H. (2011). El estudio de caso: Teoría y práctica. Madrid: Morata.

Stake, R. E. (2007). Investigación con estudio de casos. Madrid: Morata.

Vavrus, M. (2009). Sexuality, schooling, and teacher identity formation: A critical pedagogy for teacher education. Teaching and Teacher Education, 25, 383-390.

Wittig, M. (2006). El pensamiento heterosexual y otros ensayos. Barcelona: Egales.

Wolcott, H. F. (1994). Transforming qualitative data: description, analysis and interpretation. Londres: Sage.

Woyshner, C. (2002). Political history as women's history: toward a more inclusive curriculum. Theory and Research in Social Education, 30(3), 354-380. 


\section{Panta Rei}

PANTA REl es una revista digital de investigación orientada a la Historia y la Didáctica de la Historia. Su principal objetivo es la transmisión del conocimiento científico, dando una oportunidad también a los jóvenes investigadores que quieren abrirse camino en el estudio de las ciencias humanas y sociales. Se compone de estudios originales relacionados con la disciplina histórica así como su didáctica y difusión. Las diferentes secciones que componen la revista son: artículos de investigación, entrevistas a profesionales, recensiones de monografías de actualidad y crónicas de congresos o eventos científicos relevantes.

Todos los artículos publicados son objeto de un proceso de revisión a cargo de un mínimo de dos evaluadores, que se consideran expertos en el ámbito temático del artículo propuesto. Nuestro deseo es poder ofrecer unos contenidos rigurosos, de calidad y de interés.

El CEPOAT (Centro de Estudios del Próximo Oriente y la Antigüedad Tardía de la Universidad de Murcia) es la institución encargada de la coordinación y gestión de la revista, desde donde anualmente se lanzará la convocatoria para aquellos que estén interesados en publicar sus trabajos, siempre relacionados con la Historia y la Didáctica de la Historia.

PANTA REI is a digital journal focused on History and Teaching History. Its main objective is the transmission of scientific knowledge by giving also an opportunity to young researchers who want to make their way in the study of human and social sciences. It is composed by original studies related to History, as well as its didactics and promotion. The different sections of this journal are: research articles, interviews to professionals, recensions on monographs about current issues and reports about congresses or relevant scientific events.

All the articles published are subject to a revision process carried out by a minimum of two reviewers who are considered to be experts in the field of the article proposed. Our wish is to offer rigorous contents with quality and being of interest to the reader.

CEPOAT (Centre of Studies of the Middle East and Late Antiquity of the University of Murcia) is the institution in charge of the coordination and management of this journal. This is the centre from where the call for papers will be launched annually for all the people interested in publishing their papers, always related to History and Teaching History. 


\section{Normas de publicación}

El autor se compromete a enviar trabajos originales, que no se encuentren publicados en otras revistas ni en otros idiomas. Así mismo, el mismo artículo no podrá ser presentado en otras revistas mientras dure el proceso de evaluación.

\section{Envío y presentación de originales}

Las normas de edición y forma de envío de artículos a la revista se pueden consultar en https://revistas.um.es/pantarei/

Para la redacción de los trabajos se tendrá en cuenta el Manual de la American Psychological Association, en su $7 .^{a}$ edición. La extensión máxima de los trabajos será de 25 páginas. La revista acepta originales escritos en español o inglés.

\section{Proceso de valoración y evaluación}

Una vez recibidos los trabajos, la Revista realizará una primera valoración. Si el trabajo enviado se ajusta a las normas de presentación propuestas, la temática es coincidente con la línea editorial de la revista y posee la calidad científica necesaria, será remitido al consejo asesor para una primera evaluación. Si no es así en este primer paso se puede rechazar directamente los documentos que incumplan claramente la línea editorial.

Será el Consejo Asesor quien indique a la revista la originalidad, relevancia, estructura, redacción, aparato bibliográfico, etc. del trabajo enviado y, para ello, se designará a dos revisores expertos externos que evaluarán cada uno de los trabajos, que pueden formar parte (o no) de este Consejo Asesor. La selección de los revisores se ajustará a la temática y características metodológicas del trabajo. El nombre y filiación de los autores serán eliminados del trabajo para su revisión, así como los revisores actuarán de manera anónima y confidencial.

Los revisores deberán rellenar un informe de evaluación que centrará su atención en aspectos tales como características formales, originalidad y novedad de los trabajos, relevancia de las propuestas y los resultados, calidad metodológica y validez científica.

Una vez terminado el proceso se decidirá la aceptación o no de los mismos y su publicación en el número que sea pertinente, así como las modificaciones susceptibles de ser realizadas para su final publicación. Dicha notificación se enviará únicamente por correo electrónico, en un plazo máximo de seis meses. 


\section{Publishing rules}

The author is committed to submit original papers not having been published in other reviews or in other languages. In this way, it is not allowed for the same paper to be presented in other reviews during the evaluation process.

\section{Submission and presentation of originals}

The editing rules and the guidelines for the submission of papers can be consulted at https://revistas.um.es/pantarei/

The seventh edition of the Manual of the American Psychological Association will be taken into account for the writing of the papers. The length of the submitted papers will not exceed the 25 pages. The journal accepts originals written in Spanish or English.

\section{Examination and assessment process}

The Journal will submit the papers to a first examination once received. If the paper follows the presentation guidelines, the subject agrees with the editorial line of this journal, and possess the scientific quality required, it will be sent to the advisory council for a first assessment. If not, the documents which clearly fail to complete the editorial line may be rejected straightaway in this first step.

The Advisory Council will indicate the originality, relevance, structure, writing, bibliography, etc. of the text to the journal; for this purpose, two outside experts will be designated to review the papers; these experts can be (or not) part of this Advisory Council. The selection of the experts will adjust to the subject and methodological characteristics of the paper. Name and affiliation of the author will be eliminated from the text for its review, in this way experts will act anonymously and confidentially.

The experts will fill out an assessment report which will focus on aspects such as formal characteristics, originality and novelty of the papers, relevance and results of the proposal, methodological quality and scientific validity.

Once the process is finished, the acceptance or not of the papers and its publication in the corresponding edition will be decided, as well as the modifications that may be done for its final publication. This notification will be sent by email within 6 months maximum. 


\section{cepoAt edit.um}

